\title{
Multidrug resistant Acinetobacter baumannii: a descriptive study in a city hospital
}

\author{
Lemuel L Dent*1 ${ }^{* 1}$ Dana R Marshall ${ }^{+1}$, Siddharth Pratap ${ }^{\dagger 2}$ and Robert B Hulette ${ }^{+3}$
}

\begin{abstract}
Background: Multidrug resistant Acinetobacter baumannii, (MRAB) is an important cause of hospital acquired infection. The purpose of this study is to determine the risk factors for MRAB in a city hospital patient population.

Methods: This study is a retrospective review of a city hospital epidemiology data base and includes 247 isolates of Acinetobacter baumannii (AB) from 164 patients. Multidrug resistant Acinetobacter baumannii was defined as resistance to more than three classes of antibiotics. Using the non-MRAB isolates as the control group, the risk factors for the acquisition of MRAB were determined.

Results: Of the $247 \mathrm{AB}$ isolates $72 \%$ (177) were multidrug resistant. Fifty-eight percent (143/247) of isolates were highly resistant (resistant to imipenem, amikacin, and ampicillin-sulbactam). Of the 37 patients who died with Acinetobacter colonization/infection, 32 (86\%) patients had the organism recovered from the respiratory tract. The factors which were found to be significantly associated ( $p \leq 0.05$ ) with multidrug resistance include the recovery of AB from multiple sites, mechanical ventilation, previous antibiotic exposure, and the presence of neurologic impairment. Multidrug resistant Acinetobacter was associated with significant mortality when compared with sensitive strains $(p \leq 0.01)$. When surgical patients $(\mathrm{N}=75)$ were considered separately, mechanical ventilation and multiple isolates remained the factors significantly associated with the development of multidrug resistant Acinetobacter. Among surgical patients 46/75 (61\%) grew a multidrug resistant strain of $A B$ and $37 / 75$ (40\%) were resistant to all commonly used antibiotics including aminoglycosides, cephalosporins, carbepenems, extended spectrum penicillins, and quinolones. Thirty-five percent of the surgical patients had AB cultured from multiple sites and $57 \%$ of the Acinetobacter isolates were associated with a co-infecting organism, usually a Staphylococcus or Pseudomonas. As in medical patients, the isolation of Acinetobacter from multiple sites and the need for mechanical ventilation were significantly associated with the development of MRAB.
\end{abstract}

Conclusions: The factors significantly associated with MRAB in both the general patient population and surgical patients were mechanical ventilation and the recovery of Acinetobacter from multiple anatomic sites. Previous antibiotic use and neurologic impairment were significant factors in medical patients. Colonization or infection with MRAB is associated with increased mortality.

\section{Background}

Acinetobacter baumannii, found ubiquitously in the environment, is an aerobic gram negative rod which is a nonfermenter of glucose. When stationary, $A B$ appears as a coccobacillus, however during growth it takes on a rod form. Multidrug resistant Acinetobacter baumannii is an important cause of hospital acquired infection and has

\footnotetext{
* Correspondence: Ident@mmc.edu

1 Department of Surgery, Meharry Medical College, Nashville, USA + Contributed equally

Full list of author information is available at the end of the article
}

been shown in some studies to increase mortality and length of stay [1].

Multidrug Resistant Acinetobacter baumannii is often associated with co-infection by other virulent pathogens. Thus it is difficult to determine its attributable mortality. Though MRAB is considered to be a hospital acquired infection, patients occasionally present with community acquired colonization of chronic wounds. In order to provide timely and proper antibiotic therapy it is important to know the characteristics of those patients with colonization and invasive infection with MRAB. The purpose of this study is to determine the resistance patterns of $A B$ in 
a city hospital and to examine the risk factors for colonization/infection in surgical patients. The source of infection and the prevalence of co-infecting pathogens will also be investigated.

\section{Methods \\ Setting}

This research was approved by Institutional Review Board of Meharry Medical College. The Nashville General Hospital (NGH) is a 200 bed teaching tertiary safety net hospital which serves a population of 1.5 million people. NGH also serves as a major provider of inpatient care for the Tennessee Department of Corrections. The medi$\mathrm{cal} /$ surgical intensive care unit has 18 beds and is a combined general medical and surgical semi-closed unit.

\section{Study design}

This study is a retrospective review of a city hospital epidemiology data base and includes 247 isolates of AB from 164 patients. For the period 2006 through 2008 a retrospective chart review was performed on all patients with $\mathrm{AB}$ isolates. The patients were identified through the hospital infection control data base. Documented patient demographics and potential risk factors included diagnosis, length of stay, patient location, age, sex, race, previous institutionalization, previous antibiotic use, mechanical ventilation, tracheostomy, and underlying co-morbidity. The source of the AB isolates was also noted as well as the patient outcome.

Previous institutionalization included prior hospitalization, nursing home residency, or incarceration within 90 days of a positive $A B$ culture date. Prior antibiotic use was noted for those patients who received an antibiotic within one month of the $\mathrm{AB}$ isolation. The resistance patterns of all isolates were included in the analysis; however patients with more than one isolate were counted only once. The source of the $\mathrm{AB}$ isolate is the anatomic site where the culture was obtained.

\section{Identification and characterization of MRAB}

The identification of the isolates as Acinetobacter and susceptibility testing of those isolates was performed using a Siemens (formerly Dade Behering) microscan system, which is FDA approved for this use in clinical laboratories. Testing was performed according to manufacturer specifications for this instrument and was done in accordance with NCCLS recommended practices. This instrument makes use of broth microdilution methodology to determine resistance. An isolate was deemed pan-sensitive if it was sensitive to all commonly tested antibiotics except colistin and highly sensitive if it was sensitive to imipenem, amikacin, and ampicillin-sulbactam. The isolate was considered highly resistant if it was resistant to imipenem, amikacin, and ampicillin-sul- bactam. An isolate was classified as pan-resistant if it was resistant to all commonly used antibiotics. Multidrug resistant Acinetobacter baumannii isolates are defined as those resistant to more than three classes of antibiotics. A distinction was made between carbepenems and the noncarbepenem $\beta$-lactam antibiotics because carbapenem resistance is a sentinel event for emerging antimicrobial resistance and in itself confers high resistance. This study is designed to document the risk factors for the isolation of MRAB in our surgical patient population, thus no attempt was made to distinguish between colonization and invasive infection. The differences between groups were determined by Student's T-test for continuous data or the Fisher's exact test for categorical data (2-tailed). The significance level threshold was a $\mathrm{p}$-value of $\leq 0.05$.

\section{Results}

Multidrug resistant Acinetobacter baumannii is defined as resistance to more than three classes of antibiotics. Of the $247 \mathrm{AB}$ isolates, 177 (72\%) were multidrug resistant. More than half of the isolates, 143/247 (58\%) were resistant to imipenem, amikacin, and ampicillin-sulbactam; thus classifying these isolates as highly resistant. This is intriguing as these antibiotics were formerly very effective against AB. Only $42(17 \%)$ of the isolates were sensitive to all three of the above antimicrobial agents. Fortysix percent $(113 / 247)$ of the isolates were resistant to all commonly used antibiotics including aminoglycosides, cephalosporins, carbepenems, extended spectrum penicillins, and quinolones and therefore were classified as pan-resistant.

The sources of $\mathrm{AB}$ isolates in all patients are shown in Table 1. The major site of $A B$ isolation in this study was the respiratory tract. Due to the inconsistency of obtaining quantitative cultures it was not possible to determine infection vs. colonization, however $32 / 37$ (86\%) of all patients who died had a positive isolate recovered from the respiratory tract. Among the positive wound isolates the majority were from chronic diabetic wounds, amputation sites, and decubitus ulcers. Using the non-MRAB isolates as the control group, the risk factors for the acquisition of MRAB were determined (Table 2). The factors which were significantly associated with multidrug resistance include the recovery of Acinetobacter from multiple sites, mechanical ventilation, previous antibiotic use, and the presence of co-morbidity (especially neurologic impairment). Diabetes mellitus and the previous use of quinolones trended toward significance.

Forty six percent of the total patients (75/164) were admitted to the surgical service and the analysis appears in Table 3. The sites of $\mathrm{AB}$ isolation in surgical patients were wounds $36(47.4 \%)$, respiratory tract 17 (23\%), urinary tract $14(18 \%)$, blood $5(7 \%)$ and vascular catheters 3 (4\%). Thirty-five percent of the surgical patients had AB 
Table 1: Sources of 247 multi-resistant Acinetobacter baumannii isolates

\begin{tabular}{lcc}
\hline & $\mathbf{N}$ & $\%$ \\
\hline Sputum & 77 & 31 \\
\hline Urine & 40 & 16 \\
\hline Extremity Wounds & 32 & 13 \\
\hline Other & 30 & 12 \\
\hline Blood & 26 & 10 \\
\hline CVP Catheter & 23 & 9 \\
\hline Decubitus Ulcer & 19 & 8 \\
\hline
\end{tabular}

cultured from multiple sites and $57 \%$ percent of the $A B$ isolates in surgical patients were associated with a coinfecting organism, usually a Staphylococcus or Pseudomonas (Table 4). While the finding of Acinetobacter in multiple sites was associated with increased likelihood of multidrug resistance ( $\mathrm{p} \leq 0.01)$, no significant association was found for co-infecting organisms.

Among surgical patients 46/75 (61\%) of isolates were MRAB and 37/75 (40\%) were resistant to all commonly used antibiotics. The majority of surgical patients with positive $A B$ isolates $(69 \%)$ had been institutionalized as inpatients, nursing home residents, or inmates within 90 days prior to the positive AB culture. Peculiar to this hospital population, 18 (24\%) of surgical patients had been incarcerated. Three patients (4\%) had Acinetobacter recovered from chronic wounds without a history of prior institutionalization. Twenty six surgical patients (35\%) required mechanical ventilation or tracheostomy. Mechanical ventilation $(\mathrm{p}<0.01)$ was significantly associated with the development of MRAB.

The majority (92\%) of surgical patients with $\mathrm{AB}$ colonization received antibiotics within 30 days of the positive culture. The most frequent antibiotics prescribed were non-carbepenem $\beta$-Lactams (39\%), and flouroquinolones (27\%), aminoglycosides (15\%), and carbepenems (13\%). Overall prior exposure to antibiotics was not a significant risk factor in the development of MRAB in this group of surgical patients, however the previous use of non-carbepenem $\beta$-lactams trended toward significance $(\mathrm{p}=$ 0.07).

A co-morbidity was present in $63(84 \%)$ of surgical patients but overall was not a risk factor for MRAB in this study $(\mathrm{p}=0.32)$. The most frequent co-morbidity associated with MRAB in this study is diabetes mellitus 27/75
(36\%), followed by chronic obstructive pulmonary disease (12\%), neurological impairment (9\%), and cardiovascular disease (8\%).

With regard to outcome, $41 \%$ of patients were discharged to home, $32 \%$ were discharged to nursing homes or transferred to other inpatient facilities, and 16\% died. The mean length of stay for sensitive and multidrug resistant Acinetobacter (32 vs. 29 days) was not significantly different ( $p>0.05)$. Of the surgical deaths, all patients had MRAB and 9/12(75\%) were colonized or infected with pan-resistant $A B$. Overall, the presence of co-infection with other organisms did not contribute significantly to mortality $(p=0.75)$. The only organism that approached significance with respect to affecting survival was Pseudomonas aeruginosa $(\mathrm{p}=0.07)$.

\section{Discussion}

Acinetobacter baumannii has become an important pathogen in recent years and has been shown to increase morbidity and mortality [1-3]. The definition of MRAB varies in the literature, but several authorities consider an isolate to be multidrug resistant if it is resistant to three or more classes of antibiotics [4]. Resistant $A B$ is a significant problem as seen in this study where $72 \%$ of isolates were considered multidrug resistant and $58 \%$ were resistant to imipenem, amikacin, and ampicillin-sulbactam, formerly very effective antibiotics. Nearly half (46\%) of all isolates were resistant to all commonly used antibiotics including aminoglycosides, cephalosporins, carbepenems, extended spectrum penicillins, and quinolones. A distinction was made between carbepenems and the noncarbepenem $\beta$-lactam antibiotics because carbapenem resistance is a sentinel event for emerging antimicrobial resistance and in itself confers high resistance and therapeutic challenges [5].

The factors associated with the isolation of $A B$ in our combined medical and surgical patient groups include mechanical ventilation, previous antibiotic therapy, comorbidity, especially neurologic impairment, and multiple Acinetobacter isolates. The significant factors for $M R A B$ in the surgical group were mechanical ventilation, multiple isolates, and neurologic impairment. These findings are consistent with other reports [6-9]. The most frequent antibiotics prescribed in this study population were $\beta$-lactams, followed by flouroquinolones, aminoglycosides, and carbepenems. The prior exposure to quinolones trended toward significance which is documented in previous reports [10].

Several investigators have found an association of MRAB with co-morbidities [7]. Our patients had significant rates of diabetes mellitus, cardiovascular disease, chronic obstructive lung disease and neurological impairment. A co-morbid condition was found in $84 \%$ of our surgical cohort, however only neurologic impairment 
Table 2: Risk factors for 247 isolates of multi-resistant Acinetobacter baumannii (all patients, $\mathbf{N}=164$ )

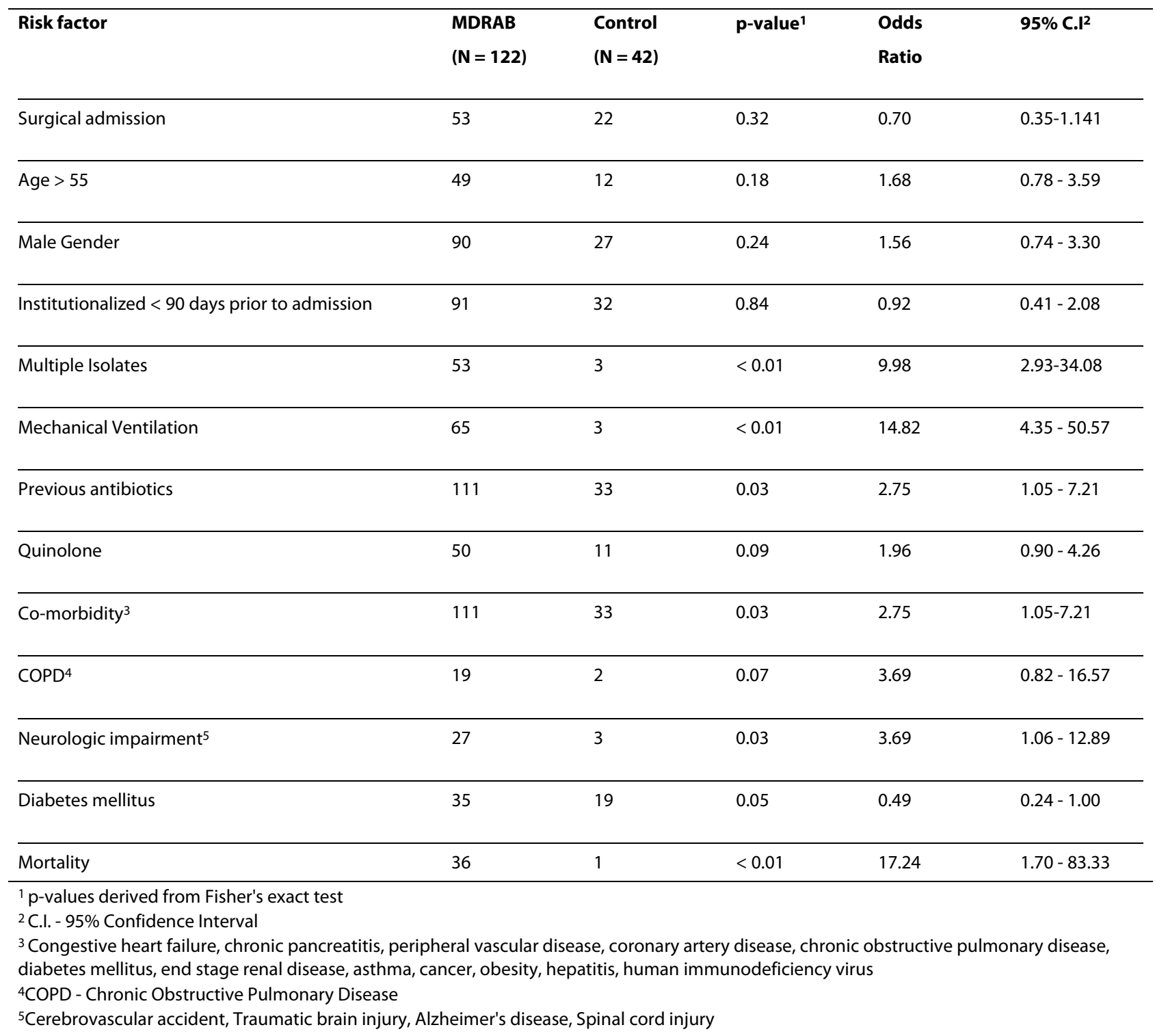

achieved significance. Neurologic injury, in particular paraplegia has been shown to be associated with the development of resistant Acinetobacter [11]. Our neurologically impaired population had a high prevalence of chronic wounds such as decubitus ulcers and most were nursing home residents. This most likely explains the significant increased risk of resistant Acinetobacter in this group.

Chronic obstructive pulmonary disease and diabetes showed a trend toward significant associations with the development of multidrug resistant Acinetobacter. It is well accepted that patients with chronic lung disease are at increased risk of airway colonization and pneumonia, especially when they require intubation [12]. Additionally, intubated patients with chronic pulmonary disease are often treated with prophylactic antibiotics which increase the risk of resistance. The association with diabetes is most likely related to the high prevalence of chronic diabetic lower extremity wounds in this patient population.

As in several other studies, this descriptive study attests to the ability of $\mathrm{AB}$ to colonize/infect soft tissue and bone $[13,14]$. Nearly half (47\%) of our surgical patients had resistant $\mathrm{AB}$ in chronic diabetic wounds, amputation sites, or decubitus ulcers. Several our patients had multiple positive cultures of their wounds over a period of several months. These patients are chronic carriers of resistant $A B$ who may develop life threatening invasive infection or serve as portals of entry of resistant $A B$ into the hospital. Whether routine surveillance and isolation 
Table 3: Risk Factors for multi-resistant Acinetobacter baumannii in surgical patients $(\mathbf{N}=\mathbf{7 5})$

\begin{tabular}{|c|c|c|c|c|c|}
\hline Risk factor & $\begin{array}{l}\text { MDRAB }^{1} \\
(\mathrm{~N}=51)\end{array}$ & $\begin{array}{l}\text { Control } \\
(\mathrm{N}=24)\end{array}$ & p-value ${ }^{2}$ & $\begin{array}{l}\text { Odds } \\
\text { Ratio }\end{array}$ & $95 \%$ C.I. ${ }^{3}$ \\
\hline Age $>55$ & 19 & 9 & 0.37 & 1.56 & $0.59-4.17$ \\
\hline Male Gender & 33 & 19 & 0.57 & 1.34 & $0.49-3.63$ \\
\hline Institutionalized $<90$ days prior to admission & 32 & 20 & 0.96 & 1.03 & $0.38-2.82$ \\
\hline Multiple Isolates & 22 & 5 & $<0.01$ & 4.40 & $1.43-13.54$ \\
\hline Mechanical Ventilation & 22 & 4 & $<0.01$ & 5.73 & $1.72-19.09$ \\
\hline Previous Antibiotics & 41 & 25 & 0.73 & 1.31 & $0.32-5.35$ \\
\hline Co-infection & 26 & 8 & 0.64 & 1.09 & $0.76-1.56$ \\
\hline Co-morbidity & 41 & 23 & 0.32 & 2.14 & $0.59-7.79$ \\
\hline Mortality & 12 & 0 & 0.01 & 1.85 & $1.48-2.33$ \\
\hline
\end{tabular}

of these patients may help to prevent outbreaks is unclear [11].

Previous institutionalization (hospital, prison, or nursing home) did not reach the level of significance ( $\mathrm{p}=$ 0.07 ) as a factor in the development of MRAB in this study. The reason for this finding is unclear since several studies have documented higher rates of MRAB in patients with a history of prior institutionalization [8]. Our patient population is unusual due to the large number $(24 \%)$ of incarcerated individuals. Three patients (4\%) had multidrug resistant Acinetobacter despite having no history of being institutionalized. The existence of community acquired MRAB strains would present significant challenges to infection control.

As seen in Tables 1 and 2, MRAB was associated with a significant $(\mathrm{p}<0.01)$ increase in mortality. An increase in mortality for ventilator associated Acinetobacter pneumonia has been noted in several studies $[2,15,16]$, however other investigators have not confirmed this finding $[17,18]$. In the surgical group, a significant proportion (35\%) had positive Acinetobacter cultures from multiple sites. Multiple isolates were associated with a significant increase in mortality. The finding of resistant $\mathrm{AB}$ isolates in more than one anatomic site most likely signifies an invasive infection rather than colonization. The majority of surgical patients (57\%) had an organism other than AB identified on culture, usually a Staphylococcus or Pseudomonas. Co-infection with non-Acinetobacter organisms did not lead to a significant increase in mortality. Pseudomonas was the only organism that approached significance with respect to mortality $(p=0.07)$

There was no significant difference in the length of stay between patients with sensitive isolates vs. multidrug resistant Acinetobacter. This finding was somewhat surprising given that some studies have shown an increase length of stay for resistant strains. The length of stay in patients with resistant organisms may be confounded by their increased mortality. The mortality rate in surgical patients was $16 \%$ and all had resistant Acinetobacter. Most of the surgical patients who died (75\%) grew panresistant strains which were resistant to all commonly used antibiotics. Underlying co-morbidities and coinfecting organisms did not significantly impact mortality in this study, whereas the presence of a multidrug resistant Acinetobacter was associated with increased mortality (OR 1.6, 95\% C.I 1.33-1.96). This suggests that colonization/infection with MRAB is a marker for severe illness or that the organism itself is responsible for poor outcome.

This study is limited because it does not identify patients with true infections versus those that are colonized. The goal was to determine the impact of MRAB in 
Table 4: Co-infecting organisms in 75 surgical patients

\begin{tabular}{lcc}
\hline Organism & N & $\%$ \\
\hline MRSA $^{1}$ & 18 & 24 \\
\hline MSSA $^{2}$ & 25 & 33 \\
\hline Pseudomonas aeruginosa & 11 & 14 \\
\hline Proteus mirabilis & 6 & 8 \\
\hline Escherichia coli & 5 & 7 \\
\hline Enterococcus faecalis & & \\
\hline Enterobacter cloacae & 5 & 7 \\
\hline Staphylococcus epidermidis & 5 & 7 \\
\hline Klebsiella pneumoniae & 4 & 5 \\
\hline 1Methicillin resistant Staphylococcus aureus & & 5 \\
\hline Methicillin sensitive Staphylococcus aureus & & \\
\hline
\end{tabular}

our hospital. The mortality rate in this study of $16 \%$ is consistent with a similar study reported by Mahgoub [9]. Of note is that the rate of MRAB in our hospital was 122/ 164 (75\%) which is higher than reported elsewhere [19]. The cause of the high rate of multi-resistance is not known and our study design does not enable us to answer this question. We are not certain what role our infection control procedures had on the high resistance rate. Standard infection control practices were followed (hand hygiene, personal protective equipment, environmental control, isolation, etc). Most of the resistant cases (75\%) occurred in the ICU, however antibiotic susceptibilities and the anatomical sources varied greatly. We speculate that since different strains were involved, it is unlikely that a breakdown in infection control was the major cause of the high rate of resistance. Initially, the multiresistant isolates were confirmed by an independent laboratory. Thus, we are confident concerning our determination of the antibiotic susceptibilities. We are planning a future study to determine the DNA fingerprinting of several of the more common resistant phenotypes in our hospital.

\section{Conclusions}

The factors associated with multidrug resistant isolates in this study were mechanical ventilation, previous antibiotic use, neurologic impairment, and the recovery of Acinetobacter from more than one anatomic site. This study also suggests that infection or colonization with $M R A B$ is associated with increased mortality. The increasing presence of MRAB in wounds is particularly problematic in the surgical patient. Chronic wounds such as amputation stumps, decubitus ulcers, and diabetic wounds are portals of entry of Acinetobacter into the surgical intensive care unit. A small number of patients present with community acquired multidrug resistant strains, thus routine surveillance may be useful to guide hospital infection control measures. Vigilance is needed by the surgical team to prevent outbreaks of this opportunistic and deadly pathogen.

\section{Competing interests \\ The authors declare that they have no competing interests.}

\section{Authors' contributions}

LD designed the study, collected and analyzed the data, and drafted the manuscript. DM assisted with study design, collection of data, revised and approved the manuscript. SP provided statistical support, assisted with study design, revised and approved manuscript. BH provided culture data, data interpretation, reviewed and approved the manuscript.

\section{Acknowledgements}

Data analyses were performed through the use of the Meharry Medical College Microarray and Bioinformatics Core, which is supported in part by $\mathrm{NIH}$ grants G12RR03032-19 and P20RR011792.

Funding for this activity was made possible (in part) by 2P20MD000516-05A1 from the National Center on Minority Health and Health Disparities. The views expressed do not necessarily reflect the official policies of the Department of Health and Human Services; nor does mention by trade names, commercial practices, or organizations imply endorsement by the U.S. Government.

\section{Author Details}

'Department of Surgery, Meharry Medical College, Nashville, USA, ${ }^{2}$ Microarray and Bioinformatics Core, Meharry Medical College, Nashville, USA and ${ }^{3}$ Department of Infection Control, Nashville General Hospital, Nashville, USA

Received: 19 November 2009 Accepted: 7 July 2010

Published: 7 July 2010

\section{References}

1. García-Garmendia JL, Ortiz-Leyba C, Garnacho-Montero J, JiménezJiménez FJ, Monterrubio-Villar J, Gili-Miner M: Mortality and the increase in length of stay attributable to the acquisition of Acinetobacter in critically ill patients. Crit Care Med 1999, 27(9):1794-1799.

2. Falagas M E, Rafailidis PI: Attributable mortality of Acinetobacter baumannii: no longer a controversial issue. Critical Care 2007, 11(3):134-136.

3. Jamulitrat $S$, Arunpan P, Phainuphong P: Attributable mortality of imipenem-resistant nosocomial Acinetobacter baumannii bloodstream infection. J Med Assoc Thai 2009, 92(3):413-419.

4. Falagas ME, Koletsi PK, Bliziotis IA: The diversity of definitions of multidrug-resistant (MDR) and pandrug-resistant (PDR) Acinetobacter baumannii and Pseudomonas aeruginosa. J Med Microbio/ 2006, 55(Pt 12):1615-1617.

5. Kluytmans-Vandenbergh M, Kluytmans J, Voss A: Dutch guideline for preventing nosocomial transmission of highly resistant microorganisms (HRMO). Infection 2005, 5/6:309-313.

6. Seifert H, Strate A, Pulverer G: Nosocomial bacteremia due to Acinetobacter baumannii: Clinical features, epidemiology, and predictors of mortality. Medicine 1995, 74(6):340-349.

7. Baran G, Erbay A, Bodur H, Ongürü P, Akinci E, Balaban N, Cevik MA: Risk factors for nosocomial imipenem resistant Acinetobacter baumannii infections. Int J Infect Dis 2008, 12(1):16-21. 
8. Falagas ME, Kopterides P: Risk factors for the isolation of multidrugresistant Acinetobacter baumannii and Pseudomonas aeruginosa: a systemic review of the literature. J Hosp Inf 2006, 64(1):7-15.

9. Mahgoub S, Ahmed J, Glatt AE: Underlying characteristics of patients harboring highly resistant Acinetobacter baumannii. Am J Infect Control 2002, 30(7):386-390.

10. Villers D, Espaze E, Coste-Burel M, Giauffret F, Ninin E, Nicolas F, Richet $H$. Nosocomial Acinetobacter baumannii infections: Microbiological and clinical epidemiology. Ann of Int Med 1998, 129(3):182-189.

11. Maragakis LL, Tucker MG, Miller RG, Carroll KC, Perl TM: Incidence and prevalence of multidrug-resistant Acinetobacter using targeted active surveillance cultures. JAMA 2008, 299(21):2513-2514.

12. Nseir S, Di Pompeo C, Cavestri B, Jozefowicz E, Nyunga M, Soubrier S, Roussel-Delvallez M, Saulnier F, Mathieu D, Durocher A: Multiple-drugresistant bacteria in patients with severe acute exacerbation of chronic obstructive pulmonary disease: Prevalence, risk factors, and outcome. Crit Care Med 2006, 34(12):2959-2966

13. Davis KA, Moran KA, McAllister CK, Gray PJ: Multi-drug-resistant Acinetobacter extremity infections in soldiers. Emerg Inf Dis 2005 11(8):1218-1224

14. Maragakis LL, Cosgrove SE, Song X, Kim D, Rosenbaum P, Ciesla N Srinivasan A, Ross T, Carroll K, Perl TM: An Outbreak of multidrugresistant Acinetobacter baumannii associated with pulsatile lavage wound treatment. JAMA 2004, 292(24):3006-3011.

15. Chastre J, Fagon JY: Ventilator-associated pneumonia. Am J Respir Crit Care Med 2002, 165:867-903.

16. Garnacho-Montero J, Ortiz-Leyba C, Jiménez-Jiménez FJ, BarreroAlmodóvar AE, García-Garmendia JL, Bernabeu-Wittell M, Gallego-Lara SL, Madrazo-Osuna J: Treatment of multidrug-resistant Acinetobacter baumannii ventilator-associated pneumonia (VAP) with intravenous colistin: A comparison with imipenem-susceptible VAP. Clin Infect Dis 2003, 36:1111-1118

17. Aybar Türkoğlu M, Topeli Iskit A: Ventilator associated pneumonia caused by high risk microorganisms: a matched case control study. Tuberk Toraks 2008, 56(2):139-149.

18. Mai MH, Tsai HC, Lee SS, Wang YH, Chen YS, Wann SR, Liu YC: Multi-drugresistant Acinetobacter baumannii in ventilator-associated pneumonia at a medical center in southern Taiwan. J Microbiol Immunol Infect 2007, 40(5):401-405

19. Peleg AY, Seifert H, Paterson D: Acinetobacter baumannii: Emergence of a Successful Pathogen. Clin Microbiol Reviews 2008:538-582.

\section{Pre-publication history}

The pre-publication history for this paper can be accessed here: http://www.biomedcentral.com/1471-2334/10/196/prepub

doi: 10.1186/1471-2334-10-196

Cite this article as: Dent et al., Multidrug resistant Acinetobacter baumannii: a descriptive study in a city hospital BMC Infectious Diseases 2010, 10:196

\section{Submit your next manuscript to BioMed Central} and take full advantage of:

- Convenient online submission

- Thorough peer review

- No space constraints or color figure charges

- Immediate publication on acceptance

- Inclusion in PubMed, CAS, Scopus and Google Scholar

- Research which is freely available for redistribution

Submit your manuscript at www.biomedcentral.com/submit
C) Biomed Central 